\section{Implications}

The protection mediated by $\mathrm{T} 2 \mathrm{R}$ signaling is likely to function against a large group of potential Gram-negative pathogens. AHLs are highly conserved molecules used by many Gram-negative bacteria to coordinate gene expression (8). Although P. aeruginosa is the best-studied AHL system and arguably the most relevant human pathogen in this group of opportunists, similar signaling systems are widespread, particularly in the marine Vibrios and Aeromonas species, as well as in common opportunists such as Acinetobacter and Burkholderia species $(11,12)$. Thus, T2R signaling would be expected to contribute to innate immune defenses against a number of potential pathogens that are associated with respiratory infection.

Given the interest in identifying genes that confer increased risk or resistance to specific diseases, would the identification of a patient's T2R38 genotype be clinically useful? In those with known increased susceptibility to respiratory infection, such as those with cystic fibrosis or COPD, or even patients in intensive care units expected to require assisted ventilation, Gram-positive as well as Gram-negative infections are fre- quent, and it would be difficult to imagine a scenario in which the T2R genotype would affect therapy that is usually based on bacterial culture results. However, in selected patient groups, such as those with chronic sinusitis, it should be possible to design a prospective study to determine whether the T2R38 genotype can be used to direct preventative antibiotic therapy.

\section{Acknowledgments}

The author's laboratory is supported by NIH grants RO1HL079395 and HL73989.

Address correspondence to: Alice Prince, Department of Pediatrics, Columbia University, 650 West 168th Street, Black Building 418, New York, New York 10032, USA. Phone: 212.305.4193; Fax: 212.305.2284; E-mail: asp7@columbia.edu.

\footnotetext{
1. Wang X, et al. Mutation in the gene responsible for cystic fibrosis and predisposition to chronic rhinosinusitis in the general population. JAMA. 2000;284(14):1814-1819.

2. Lee RJ, et al. T2R38 taste receptor polymorphisms underlie susceptibility to upper respiratory infection. J Clin Invest. 2012;122(11):4145-4159.

3. Chandrashekar J, et al. T2Rs function as bitter taste receptors. Cell. 2000;100:703-714.

4. Brockhoff A, Behrens M, Massarotti A, Appendino G, Meyerhof W. Broad tuning of the human bitter
}

taste receptor hTAS2R46 to various sesquiterpene lactones, clerodane and labdane diterpenoids, strychnine, and denatonium. J Agric Food Chem. 2007;55(15):6236-6243

5. Meyerhof W. Elucidation of mammalian bitter taste. Rev Physiol Biochem Pharmacol. 2005;154:37-109.

6. Kim UK, Jorgenson E, Coon H, Leppert M, Risch $\mathrm{N}$, Drayna D. Positional cloning of the human quantitative trait locus underlying taste sensitivity to phenylthiocarbamide. Science. 2003; 299(5610):1221-1225.

7. Tan J, Abrol R, Trzaskowski B, Goddard WA 3rd. 3D structure prediction of TAS2R38 bitter receptors bound to agonists phenylthiocarbamide (PTC) and 6-n-Propylthiouracil (PROP). J Chem Inf Model. 2012;52(7):1875-1885.

8. Davies DG, et al. The involvement of cell-to-cell signals in the development of a bacterial biofilm. Science. 1998;280(5361):295-298.

9. Shah AS, Ben-Shahar Y, Moninger TO, Kline JN, Welsh MJ. Motile cilia of human airway epithelia are chemosensory. Science. 2009;325(5944):1131-1134.

10. Deshpande D, et al. Bitter taste receptors on airway smooth muscle bronchodilate by localized calcium signaling and reverse obstruction. Nat Med. 2010;16(11):1299-1304.

11. Anbazhagan D, Mansor M, Yan GO, Md Yusof MY, Hassan H, Sekaran SD. Detection of quorum sensing signal molecules and identification of an autoinducer synthase gene among biofilm forming clinical isolates of Acinetobacter spp. PloS One. 2012; 7(7):e36696.

12. Galloway WR, Hodgkinson JT, Bowden S, Welch M, Spring DR. Applications of small molecule activators and inhibitors of quorum sensing in Gram-negative bacteria [published online ahead of print July 6, 2012]. Trends Microbiol. doi:10.1016/ j.tim.2012.06.003

\title{
Turning a blind eye to anti-VEGF toxicities
}

\section{Susan E. Quaggin}

The Samuel Lunenfeld Research Institute, Mount Sinai Hospital, and Division of Nephrology, St. Michael's Hospital, University of Toronto, Toronto, Ontario, Canada.

\begin{abstract}
Excessive blood vessel growth is a key feature of many retinal diseases, and recently, anti-VEGF therapy has been successfully applied to treat neovascular age-related macular degeneration (AMD), diabetic macular edema, and retinal vein occlusion. In this issue of the JCI, Kurihara et al. reveal an essential role of Vegfa in maintaining choroid vasculature and cone photoreceptors, critical for central and color vision. Their findings suggest that therapeutic approaches to blocking VEGF signaling in retinal diseases might have unexpected detrimental side effects and that the development of alternative strategies might be necessary.
\end{abstract}

Anti-VEGF agents are a rapidly expanding group of therapeutic compounds developed to target pathologic angiogenesis and excessive vascular permeability in cancers and other diseases. They act by blocking the action of the VEGFA ligand or its receptors. Currently, more

Conflict of interest: The author has declared that no conflict of interest exists.

Citation for this article: $J$ Clin Invest. 2012 122(11):3849-3851. doi:10.1172/JCI65509. than 1500 clinical trials of anti-VEGF receptors are underway. The FDA has already approved anti-VEGF agents for the treatment of solid tumors, including colon, breast, and renal cell carcinoma. A number of eye diseases are also characterized by excessive blood vessel growth and permeability, including neovascular agerelated macular degeneration (AMD), diabetic retinopathy, and retinal vein occlusion (1-6). In a randomized clinical trial, patients with AMD who were treat- ed with intravitreal injections of ranibizumab (Lucentis), which inhibits VEGF ligand binding to its receptor, exhibited improvement in best corrected visual acuity (BCVA) at 12 and 24 months, compared with sham-injected controls (6). These results provided the rationale to consider using these therapies in other retinal diseases characterized by enhanced vascular permeability, such as diabetic retinopathy. In an open-label pilot study in ten patients with diabetic macular edema (DME), multiple intravitreal injections of ranibizumab reduced retinal thickness and improved BCVA at three months (7). Since this original study, seven clinical studies demonstrating positive results for DME have been published (3). While these results are very encouraging and offer potential therapeutic value to many patients, there may be reason for guarded optimism based 
A Control littermate

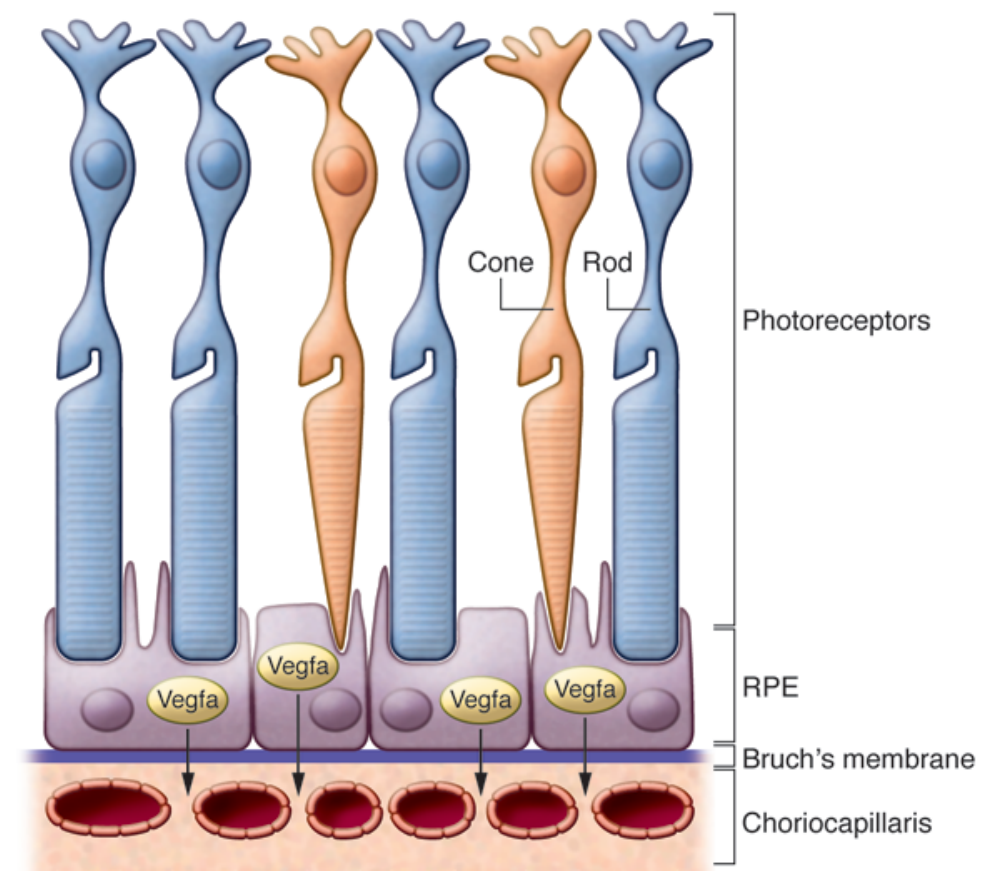

B RPE-specific inducible knockout of Vegfa

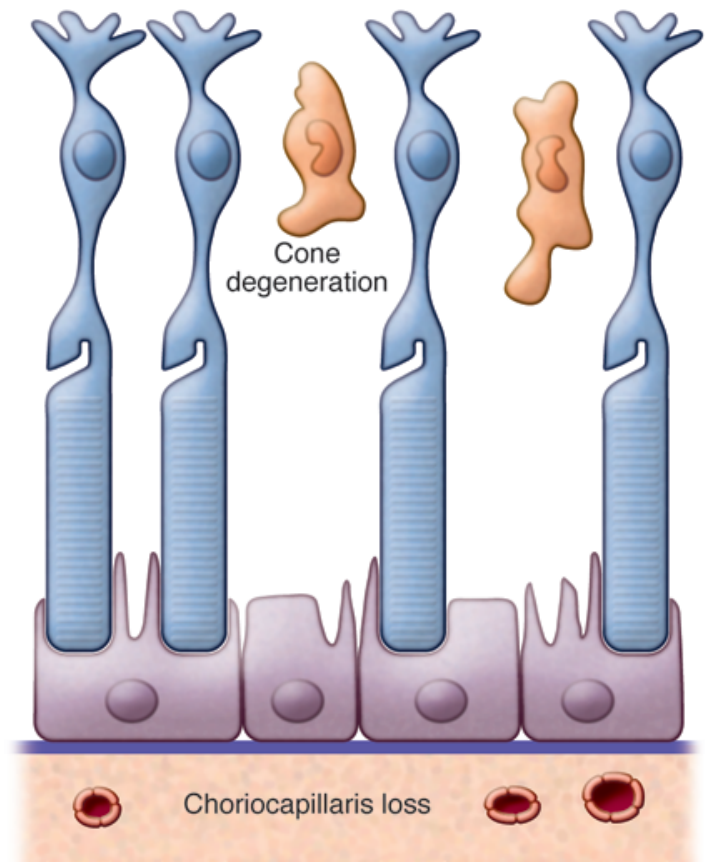

\section{Figure 1}

Vegfa produced by RPE cells is required for maintenance of the subretinal vasculature and cone photoreceptors in mice. (A) In the adult eye, retinal pigment epithelial cells produce Vegfa, which is required for maintenance of the fenestrated choriocapillaris. (B) Following RPE-specific deletion of the Vegfa gene from adult mice, the choriocapillaris rapidly disappears and is associated with death of cone photoreceptors required for central and color vision. In contrast, the rod photoreceptors are not damaged.

on a report in this issue of the JCI. Kurihara and colleagues describe dramatic and rapid loss of endothelial cells of the choriocapillaries and severe vision loss secondary to cone cell death in mice following genetic deletion of Vegfa from retinal pigment epithelial (RPE) cells, raising a note of caution regarding long-term use of these drugs (8).

\section{VEGF}

VEGFA is a trophic endothelial growth factor that promotes endothelial migration, proliferation, survival, and differentiation and enhances vascular permeability. It is often referred to as the "master angiogenic factor," since conventional knockout of the gene during embryonic development in mice results in death during midgestation due to failure of vascular formation; indeed, even loss of a single Vegfa allele during development is sufficient to result in major vascular defects and death (9). While the role of VEGFA in developmental and pathologic angiogenesis is indisputable, its role in maintaining mature, healthy blood vessels is less clear. Early studies in rodent models and preclinical studies in nonhuman primates and rabbits suggested that side effects of anti-VEGF therapies in adults might be minimal (10). However, as the number of patients treated with these agents increased, toxicities were reported $(11,12)$. In the kidney, it is now well known that VEGFA production by glomerular podocytes is required for maintenance of the glomerular microvasculature and kidney function. A small subset of patients receiving anti-VEGF therapy, as well as pregnant patients with elevated circulating levels of soluble FLT1 (an endogenous VEGFA decoy/ antagonist), develop thrombotic microangiopathy (TMA) - a disease characterized by dramatic glomerular endothelial injury (11, 13-15). In keeping with these clinical findings, podocyte-specific deletion of the Vegfa gene in mice results in renal-restricted TMA due to endothelial damage $(11,13)$.

\section{VEGF and the retina}

RPE cells share a number of characteristics with podocytes in that they are vasculature support cells for an intimately associated fenestrated endothelial bed - in this case, the choriocapillaris under the retina. Like podocytes, RPE cells constitutively express VEGFA both during development and in adulthood, suggesting an ongoing requirement for this growth factor in maintaining the adjacent choriocapillaris. Kurihara et al. determined that mice developed rapid changes in the subretinal vasculature following RPE-specific Cre-mediated deletion of Vegfa, characterized by complete loss of the choriocapillaris and marked attenuation of choroid vessels. In addition, damage to the cone photoreceptors occurred as early as three days following Cre induction (see Figure 1). Importantly, the Cre recombinase activity was induced upon addition of doxycycline, permitting assessment of the role of Vegfa in retinal and vessel maintenance rather than development. The authors suggest that the metabolically demanding cones, essential for central and color vision, may be more dependent on vascular supply than the rods, which are responsible for night vision and appeared unaffected in their genetic model (8). Alternatively, Vegfa 
may have direct neurotrophic effects on the cone photoreceptors. In keeping with this possibility, photoreceptors have been shown to express VEGF receptors (16); future studies targeting one or more of the Vegf receptors in the photoreceptor population will be required to address this possibility.

\section{Risks of VEGF antagonists in retinal disease?}

How do these findings relate to the millions of patients treated with anti-VEGF treatments for retinal disease? To date, photoreceptor damage and/or visual loss have not been widely reported in patients receiving these therapies. On the other hand, the situation may be similar to the case of kidney toxicities where only a small proportion of patients develop clinically relevant renal toxicity, while $100 \%$ of podocyte-specific knockout mice develop TMA and kidney failure (11). Complete deletion of genes is a "sledge hammer" approach, removing most if not all of the Vegfa produced. These experiments are a practical and important way to reveal functional roles for genes and pathways, but they do not necessarily recapitulate clinical circumstances. Not only is the degree of knockdown much lower with drug inhibition, but the treatment is intermittent, including drug-free periods that may allow recovery of damaged endothelium. Furthermore, it is likely that susceptibility is determined by other modifying genetic factors; identification of these will be important going forward.

In this regard, clinical studies assessing photoreceptor function and central vision, which require specialized tests such as electroretinography (ERG), have not been widely performed in assessing outcomes of anti-VEGF therapies. In addition, long-term followup will be important to assure effects were not missed in studies performed less than 24 months after treatment.

\section{New approaches?}

Given the ongoing role of VEGFA for maintenance of the retinal vasculature, are there alternative strategies to treating vascular diseases of the eye? In their paper, Kurihara and colleagues also deleted hypoxia inducible factor $1 \alpha$ (Hif1a) and hypoxia inducible factor $2 \alpha$ (Epas1) from RPE cells (8). HIFs are basic-helixloop helix transcription factors that reg- ulate VEGFA production, and similar to VEGFA, their expression is upregulated in retinal diseases. In contrast to VEGF deletion, loss of Hifla and/or Epas1 did not cause vision loss or endothelial damage in mice. However, elimination of these factors rescued pathologic angiogenesis in the laser photocoagulation model of choroidal neovascularization. The authors suggest that Hifs may be a more desirable target in eye disease, shutting down pathologic angiogenesis without damaging healthy vessels, perhaps by more modest regulation of endogenous VEGFA levels. Nonetheless, feasibility of this approach in patients and/or experimental models must be established. Although VEGFA is one of the best-known targets of the HIF pathway, endogenous levels of Vegfa were preserved in the RPE-Hifknockout mice. This suggests a dominant role for Hif-independent regulation of Vegfa or perhaps that actual deletion of the Hif allele(s) was lower than the $68 \%$ efficiency rate suggested by experiments in reporter mice (8). Combination angiostatic therapies, which include induction with an anti-VEGF agent and maintenance with a non-VEGF agent with angiostatic properties, is another possibility (17).

This study demonstrates an important functional role for Vegfa in supporting the adult subretinal vasculature, including the choriocapillaris, which is particularly important for nurturing the cone photoreceptors and maintaining central vision. This is the first documentation for a critical impact of Vegfa on the mammalian adult retina and should send a note of caution to clinicians who are treating patients with anti-VEGF agents for retinal disease. It will be important to carefully screen treated patients for detrimental long-term effects of VEGF knockdown on their vision. Future studies to identify susceptibility factors for antiVEGF toxicities in eye and other organs are also needed.

\section{Acknowledgments}

S.E. Quaggin is funded by CIHR grants M0P62931 and M0P77756, and TF grant 016002 and holds the Gabor-Zellerman Chair in Renal Research, UHN, University of Toronto.

Address correspondence to: Susan Quaggin, The Samuel Lunenfeld Research Institute, Room 5-1032, Mount Sinai Hospital,
60 Murray Street, Box 41, Toronto, Ontario M4T 2Y4, Canada. Phone: 416.586.4800, ext. 2859; Fax: 416.586.5130; E-mail: quaggin@ lunenfeld.ca.

1. Aiello LP, et al. Suppression of retinal neovascularization in vivo by inhibition of vascular endothelial growth factor (VEGF) using soluble VEGF-receptor chimeric proteins. Proc Natl Acad Sci U S A. 1995;92(23):10457-10461.

2. Campochiaro PA. Anti-vascular endothelial growth factor treatment for retinal vein occlusions. Ophthalmologica. 2012;227(suppl 1):30-35.

3. Ho AC, et al. Anti-vascular endothelial growth factor pharmacotherapy for diabetic macular edema: a report by the American Academy of Ophthalmology [published online ahead of print August 20, 2012]. Ophthalmology. doi:10.1016/ j.ophtha.2012.07.058.

4. Ip MS, et al. Anti-vascular endothelial growth factor pharmacotherapy for age-related macular degeneration: a report by the American Academy of Ophthalmology. Ophthalmology. 2008;115(10):1837-1846.

5. Pece A, Isola V, Piermarocchi S, Calori G. Efficacy and safety of anti-vascular endothelial growth factor (VEGF) therapy with intravitreal ranibizumab (Lucentis) for naive retinal vein occlusion: 1-year follow-up. Br J Ophthalmol. 2011; 95(1):56-68.

6. Rosenfeld PJ, et al. Ranibizumab for neovascular age-related macular degeneration. $N$ Engl J Med. 2006;355(14):1419-1431.

7. Chun DW, Heier JS, Topping TM, Duker JS, Bankert JM. A pilot study of multiple intravitreal injections of ranibizumab in patients with centerinvolving clinically significant diabetic macular edema. Ophthalmology. 2006;113(10):1706-1712.

8. Kurihara T, Westenskow PD, Bravo S, Aguilar E, Friedlander M. Targeted deletion of Vegfa in adult mice induces vision loss. J Clin Invest. 2012;122(11):4213-4217.

9. Carmeliet P, et al. Abnormal blood vessel development and lethality in embryos lacking a single VEGF allele. Nature. 1996;380(6573):435-439.

10. Gerber HP, et al. VEGF is required for growth and survival in neonatal mice. Development. 1999;126(6):1149-1159.

11. Eremina V, et al. VEGF inhibition and renal thrombotic microangiopathy. $N$ Engl J Med. 2008;358(11):1129-1136.

12. Frangie C, Lefaucheur C, Medioni J, Jacquot C, Hill GS, Nochy D. Renal thrombotic microangiopathy caused by anti-VEGF-antibody treatment for metastatic renal-cell carcinoma. Lancet Oncol. 2007;8(2):177-178.

13. Eremina V, et al. Glomerular-specific alterations of VEGF-A expression lead to distinct congenital and acquired renal diseases. J Clin Invest. 2003;111(5):707-716.

14. Levine RJ, et al. Circulating angiogenic factors and the risk of preeclampsia. $N$ Engl J Med. 2004;350(7):672-683.

15. Maynard SE, et al. Excess placental soluble fms-like tyrosine kinase 1 (sFlt1) may contribute to endothelial dysfunction, hypertension, and proteinuria in preeclampsia. J Clin Invest. 2003;111(5):649-658.

16. Saint-Geniez M, et al. Endogenous VEGF is required for visual function: evidence for a survival role on muller cells and photoreceptors. PLoS One. 2008;3(11):e3554

17. Dorrell MI, Aguilar E, Scheppke L, Barnett $\mathrm{FH}$, Friedlander M. Combination angiostatic therapy completely inhibits ocular and tumor angiogenesis. Proc Natl Acad Sci U S A. 2007; 104(3):967-972. 